\title{
MLLI promotes cervical carcinoma cell tumorigenesis and metastasis through interaction with $\beta$-catenin
}

This article was published in the following Dove Press journal:

OncoTargets and Therapy

28 October 2016

Number of times this article has been viewed

\author{
Rong Qiang ${ }^{1,2}$ \\ $\mathrm{Na} \mathrm{Cai}{ }^{2}$ \\ Xiaobin Wang ${ }^{2}$ \\ Lin Wang ${ }^{2}$ \\ Ke Cui ${ }^{2}$ \\ Xiang Wang' \\ $\mathrm{Xu} \mathrm{Li}$ \\ 'Center for Translational Medicine, \\ The First Affiliated Hospital of Xi'an \\ jiaotong University, ${ }^{2}$ Medical Heredity \\ Research Center, Northwest Women's \\ and Children's Hospital, Shaanxi, \\ People's Republic of China
}

\begin{abstract}
MLL protein genes encode a family of crucial transcription factors that play a key role in multiple cancer development. The functions of different MLL proteins have not been definitively studied. MLL1 is a histone methyltransferase that mediates histone H3 lysine 4, and it has been found to have aberrant expression in several tumors. However, the function of MLL1 in cervical carcinoma is little known. We used tissue analysis, cell culture experiments, and molecular profiling to investigate the mechanism of MLL1 in cervical carcinoma development. We report here that MLL1 is overexpressed in cervical carcinoma tissues and cell lines, and its overexpression is correlated with the tumor grade. Through FACScan flow cytometry assay, we found that MLL1 promotes cell proliferation by promoting the $\mathrm{G}_{1} / \mathrm{S}$ transition through transcriptional activation of CCND1 in cervical carcinoma cells. Furthermore, we utilized co-immunoprecipitation and glutathione S-transferase pull-down assays to identify $\beta$-catenin as the transcription partner for MLL1 and demonstrated that MLL1 and $\beta$-catenin act in synergy in the transcriptional activation of $C C N D 1$ in cervical carcinoma cells. In addition, transwell assay and anchorage-independent cell growth assay also revealed that MLL1 promotes metastasis of cervical carcinoma cells through interaction with $\beta$-catenin. Our study not only demonstrated a role for MLL1 in the proliferation and metastasis of cervical carcinoma cells but also revealed the interaction of MLL1 with $\beta$-catenin to play a different role.
\end{abstract}

Keywords: MLL1, cell cycle, $\beta$-catenin, metastasis

\section{Introduction}

Cervical carcinoma has become the fourth most common cancer in women in the world. ${ }^{1}$ The 5 -year overall survival of cervical cancer is $\sim 60 \%$ in all stages due to dramatic advances in screening and effective treatment. ${ }^{2}$ However, understanding the molecular pathogenic processes is necessary to identify a novel marker that may improve diagnosis and predict outcomes.

MLL1 has been reported to be associated with myelogenous leukemias or acute lymphoblastic leukemias. ${ }^{3-5}$ MLL1 is a histone methyltransferase that mediates histone $\mathrm{H} 3$ lysine $4^{6}$ and can catalyze $\mathrm{H} 3 \mathrm{~K} 4$ monomethylation, dimethylation, and trimethylation. MLL1 contains numerous conserved domains that assemble several protein complexes. MLL1 takes part in multiple crucial biological processes, such as hematopoiesis and development. ${ }^{6-9}$ However, the function of MLL1 in cervical carcinoma is still unknown.

The Wnt signaling pathway plays pivotal roles in the development of cancer and other diseases. The $\beta$-catenin functions as an important member of the canonical Wnt signaling pathway. A previous study indicated that Wnt signaling pathway works as a promoter in epithelial-mesenchymal transition and invasion/metastasis in breast
Center for Translational Medicine, The First Affiliated Hospital of Xi'an Jiaotong University, 277 West Yanta Road, Xi'an 7I006I, Shaanxi, People's Republic of China

Tel +862985324630

Email xulijiayou|3|4@yeah.net 
cancer, ${ }^{10}$ and many components of Wnt signaling pathway have been found to play a crucial role in breast carcinoma metastasis and are novel markers of poor prognosis. ${ }^{11}$ Numerous Wnt target genes are linked to oncogenesis, such as cyclin D1. Activation of cyclin D1 has been found to be correlated with poor clinical outcomes. ${ }^{12}$

In our study, we found that MLL1 was overexpressed in cervical carcinoma and that the levels of MLL1 expression were obviously correlated with tumor grades. We demonstrated that MLL1 promoted the proliferation of cervical carcinoma cells by promoting the $\mathrm{G}_{1} / \mathrm{S}$ transition and via transcriptional activation of the CCND1. In addition, we found that $\beta$-catenin interacted with MLL1 in cervical carcinoma cells and that $\beta$-catenin synergized with MLL1 in the transactivation of CCND1. Furthermore, MLL1 also facilitated metastasis of cancer cells.

\section{Materials and methods}

\section{Tissue specimens and cell lines}

Cervical carcinoma tissues were obtained from The First Affiliated Hospital of Xi'an Jiaotong University. All tissue samples were frozen at $-80^{\circ} \mathrm{C}$ until use. All human tissue experiments were approved by the ethics committee of Xi' an Jiaotong University. Normal human cervical cell lines, Ect1/E6E7, and cervical carcinoma cell lines, HeLa, SiHa, and C-33A were purchased from the American Type Culture Collection (Manassas, VA, USA). All participants provided written informed consent.

Cells were cultured in Dulbecco's Modified Eagle's Medium (DMEM; Thermo Fisher Scientific, Waltham, MA, USA) supplemented with penicillin/streptomycin $(50 \mu \mathrm{g} / \mathrm{mL})$, $10 \%$ fetal bovine serum (FBS), and $2 \mathrm{mM}$ L-glutamine at $37^{\circ} \mathrm{C}$ in $5 \% \mathrm{CO}_{2}$.

\section{Cell transfection assays}

HeLa, SiHa, and C-33A cells were transiently transfected with pcDNA3.1-MLL1 or MLL1-siRNA, using Lipofectamine 2000 transfection reagent (Thermo Fisher Scientific) according to the manufacturer's instructions. In short, cells were plated at a density of $2 \times 10^{5}$ cells in a six-well plate containing $2.5 \mathrm{~mL}$ of DMEM with penicillin/streptomycin and $10 \%$ FBS. During transfection, $3 \mu \mathrm{g}$ plasmids and $15 \mu \mathrm{L}$ polyethylenimine reagent were added in DMEM and incubated for 15 minutes at room temperature.

\section{RNA isolation and quantitative reverse transcription polymerase chain reaction assays}

TRIzol isolation reagent (Thermo Fisher Scientific) was used to extract total RNA from the cultured cells according to the manufacturer's protocol. Then, first-strand cDNA was synthesized through Reverse Transcription System (A3500; Promega Corporation, Fitchburg, WI, USA). The relative expression level of mRNA was confirmed by quantitative reverse transcription polymerase chain reaction (qRT-PCR). The PCR reaction was performed at $95^{\circ} \mathrm{C}$ for 8 minutes followed by 38 cycles at $95^{\circ} \mathrm{C}$ for 15 seconds and $57^{\circ} \mathrm{C}$ for 60 seconds. Specific primers to MLL1, CCND1, CCNE1, $p 21$, and $p 27$ were used, and GAPDH mRNA levels were used as internal control.

\section{Cell cycle analysis}

The collected cells were fixed with $70 \%$ ethanol at $-20^{\circ} \mathrm{C}$ overnight. The cells were washed twice with ice-cold PBS and $1 \mathrm{mg} / \mathrm{mL}$ RNase A (Sigma-Aldrich Co., St Louis, MO, USA) was added at $37^{\circ} \mathrm{C}$ for $30-60$ minutes and finally stained with propidium iodide for 20 minutes in the dark. FACScan flow cytometry was used to measure the DNA contents. Each experiment was performed at least three times.

\section{Chromatin immunoprecipitation assay}

Chromatin immunoprecipitation assays were performed as described previously. ${ }^{13-18} C C N D 1$ forward primer was 5'-CAGAGCCACCTCCACCTCAC-3' and CCND1 reverse primer was 5'-CACCACGCAAAGATCAAAGC-3'.

\section{Luciferase activity assay}

A total of $4 \times 10^{4}$ cells were plated in six-well plates and transfected with indicated plasmid, while the density of cells reached $\sim 70 \%-80 \%$ confluence. Transfection was carried out for almost 72 hours, using an illuminometer (Centro LB 960; Berthold Technologies, Bad Wildbad, Germany), to quantify the activities of luciferase. All experiments were performed independently for three times.

\section{Transwell assay}

A Millipore chamber (EMD Millipore, Billerica, MA, USA) was used to perform transwell assay. High invasion cervical carcinoma cell lines cell C-33A were transfected with either scrambled siRNA or MLL1-siRNA and vector or MLL1siRNA plus $\beta$-catenin expression construct, and placed $2 \times 10^{4}$ cells to the upper chamber which DMEM media was FBS-free, and added DMEM media which contain 10\% FBS in the bottom chambers. The cells were incubated with $5 \%$ $\mathrm{CO}_{2}$ for 15 hours at $37^{\circ} \mathrm{C}$, and then the cells in the upper membrane surface were removed by cotton and $0.1 \%$ crystal violet was used to stain the cells in the lower membrane surface. Finally, the cells were counted. All experiments were performed independently for three times. 


\section{Anchorage-independent growth assay}

The colony formation on soft agar was used to assess cell anchorage-independent growth ability. In brief, $2 \times$ DMEM media that contained $20 \%$ FBS and equal volumes of $1.2 \%$ agar were mixed at $37^{\circ} \mathrm{C}$ for 1 hour. The $0.6 \%$ agar acted as a base agar in six-well plates. C-33A was transfected with either scrambled siRNA or MLL1-siRNA and vector or MLL1-siRNA plus $\beta$-catenin expression construct, and $5 \times 10^{4}$ cells were suspended in $1.5 \mathrm{~mL}$ of $0.7 \%$ agar and $1.5 \mathrm{~mL}$ of $2 \times$ DMEM (with $20 \% \mathrm{FBS}$ ) and placed to each well. The cells were incubated at $37^{\circ} \mathrm{C}$ with $5 \% \mathrm{CO}_{2}$ for 15 days. Colonies were stained with $0.1 \%$ crystal violet and measured by photomicrography.

\section{Statistical analysis}

Each result was expressed as mean \pm SD. Differences were compared between adjacent normal tissues and cancer tissues through $\chi^{2}$ test. Student's $t$-test was used to compare between two groups. $P<0.05$ was considered statistically significant. All data were analyzed by SPSS 19.0 (IBM Corporation, Armonk, NY, USA; $* P<0.05, * * P<0.01)$.

\section{Results \\ MLLI is overexpressed in cervical carcinoma and correlated with tumor grade}

We first detected the expression of MLL1 in 82 cervical carcinoma tissues and adjacent normal tissues using immunohistochemistry. The results revealed that while adjacent normal tissues displayed weak MLL1 staining, cervical carcinoma tissues were strongly positive for MLL1 staining (Figure 1A).

To further explore whether the expression of MLL1 has relationship with the development and progression of cervical carcinoma, we next performed qRT-PCR analysis to detect the relative expression levels of MLL1. We defined the expression level of MLL1 in normal tissues as normal to normalize the expression of MLL1 in the tumor tissues. In 82 cervical carcinoma tissues and adjacent normal tissues that we examined, we observed a significant difference in the level of MLL1 expression. MLL1 overexpression was observed in 35\% (seven of 20) of grade I tumors, 57.1\% (16 of 28) of grade II tumors, and $73.5 \%$ ( 25 of 34 ) of grade III tumors (Figure 1B). Moreover, Western blot analysis also confirmed that MLL1
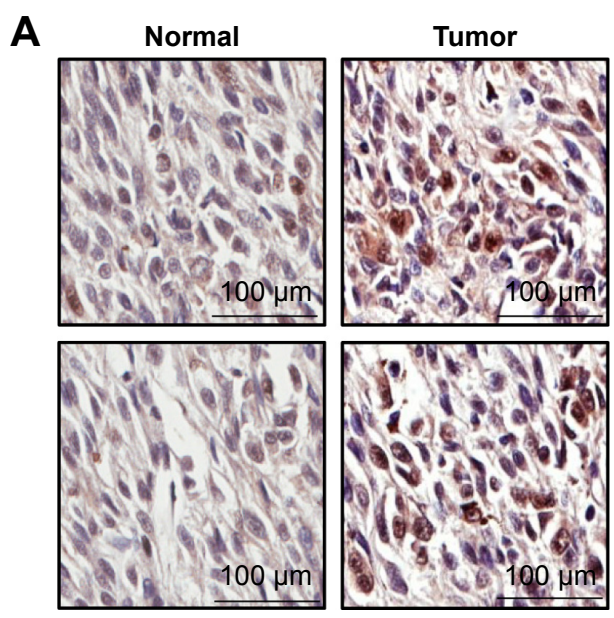

B

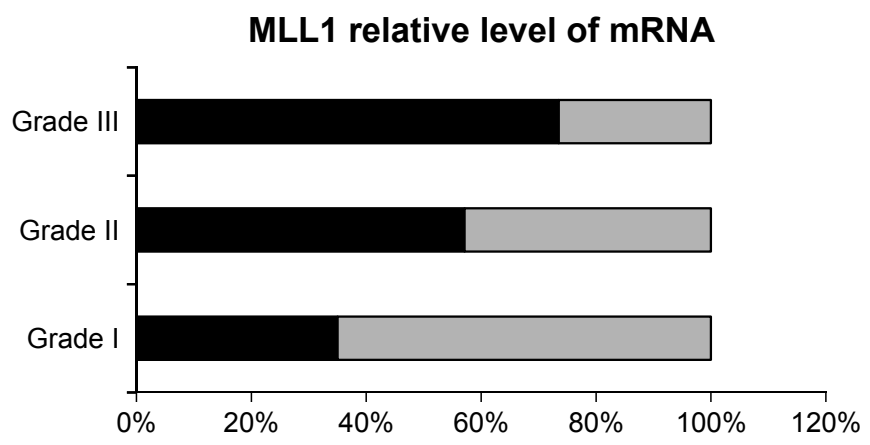

Relative expression of MLL1

MLL1 high expression $\square$ MLL1 low expression

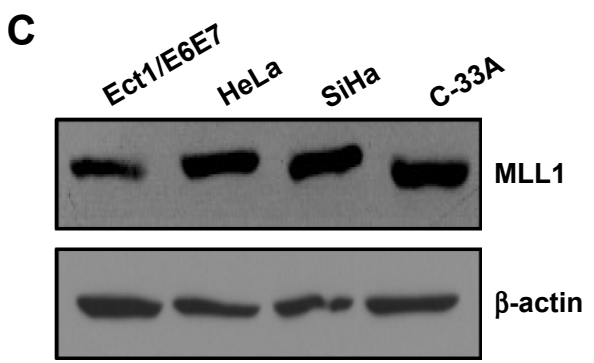

Figure I MLLI is overexpressed in cervical carcinoma and correlated with tumor grade.

Notes: (A) Representative IHC of MLLI protein expression in paraffin-embedded human cervical carcinoma tissue and adjacent normal tissue. (B) MLLI expression levels in normal and histological grades I, II, and III of cervical carcinoma. Normalized MLLI mRNA expression was measured by qRT-PCR with GAPDH expression as the internal control. (C) Western blot analysis of endogenous MLLI expression in normal human cervical cell lines and cervical carcinoma cell lines. $\beta$-actin was used as the internal control.

Abbreviations: IHC, immunohistochemistry; qRT-PCR, quantitative reverse transcription polymerase chain reaction. 
was overexpressed in cervical carcinoma cell lines, $\mathrm{SiHa}, \mathrm{HeLa}$, and C-33A, compared with normal human cervical cell lines, Ect1/E6E7 (Figure 1C). Collectively, the abovementioned data revealed that the overexpression of MLL1 might be a frequent event in human cervical carcinoma and the expression level of MLL1 is correlated with the tumor grade.

\section{MLLI promotes proliferation of cervical carcinoma cells}

We next investigated the function of MLL1 in cell proliferation. First, both loss-of-function and gain-of-function experiments were performed to explore the effect of MLL1 knockdown or overexpression on cell-cycle regulation in cervical carcinoma cells. The efficiency of MLL1 protein knockdown or overexpression in HeLa cells was detected by Western blotting (Figure 2A). In these experiments, HeLa cells were synchronized at the $\mathrm{G}_{0} / \mathrm{G}_{1}$ phase, $\mathrm{G}_{1} / \mathrm{S}$ boundary, and $\mathrm{M}$ phase (mitosis) by serum starvation, double thymidine blocking, and nocodazole blocking, respectively. ${ }^{19}$ Consequently, cell-cycle profiling was determined by FACScan flow cytometry. The results indicated that after being released from the indicated blocking, the cells with

\section{A}
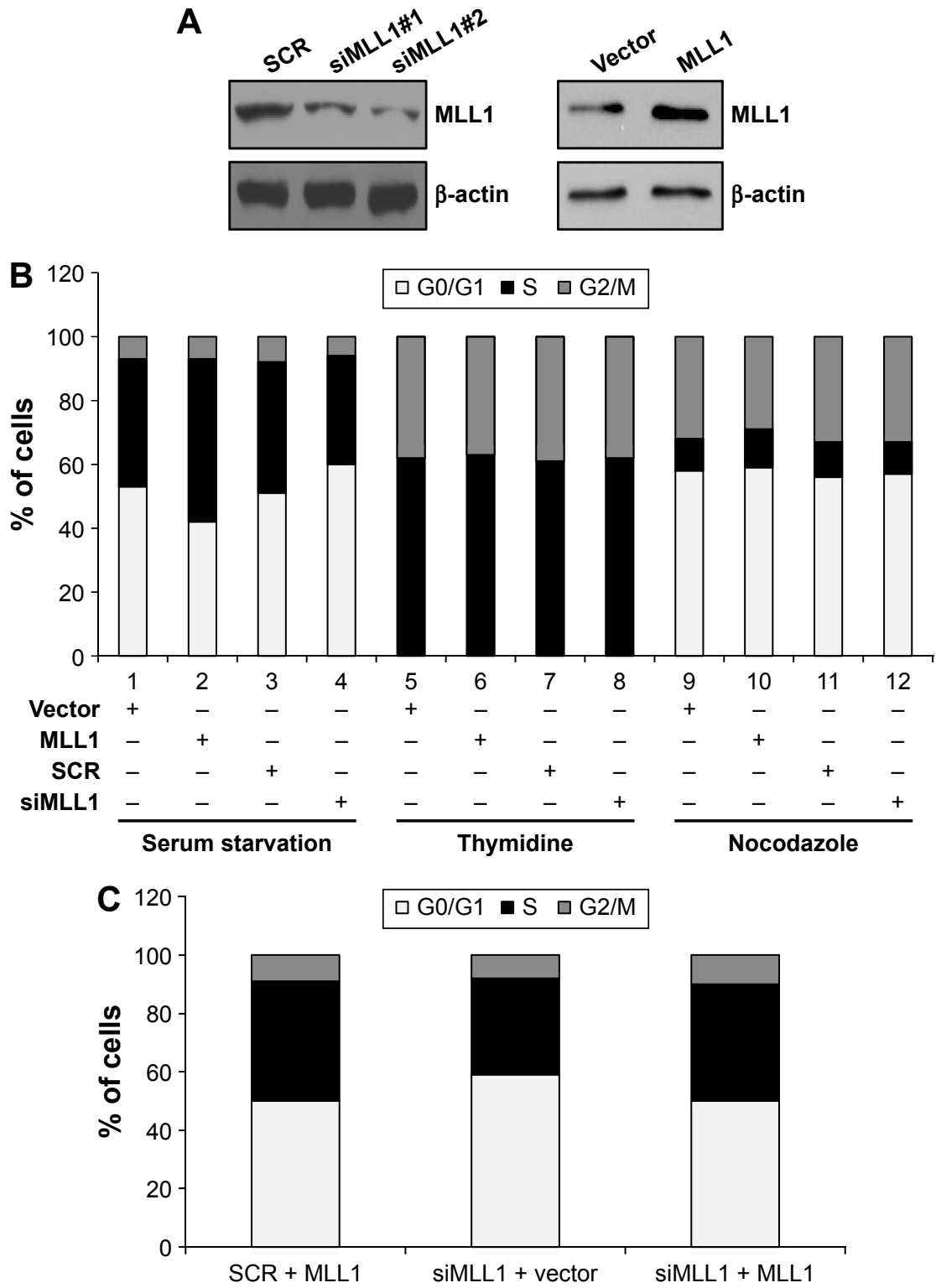

Figure 2 MLLI promotes proliferation of cervical carcinoma cells.

Notes: (A) The expression of MLLI in HeLa cells transfected with FLAG-MLLI and MLLI-siRNA was detected by Western blotting. (B) The expression of MLLI was knockdown or overexpressed in HeLa cells, and the cells were collected for cell-cycle profile analysis through flow cytometry. The percentage of cells in different cell-cycle phases are shown. (C) Serum-starved HeLa cells were treated under indicated experimental conditions after 24 hours of transfection, and the cells were released by culturing in the DMEM medium with 10\% FBS for another 18 hours. Next, the cells were collected for cell-cycle profile analysis through flow cytometry. The percentage of cells in different cell-cycle phases are shown.

Abbreviations: DMEM, Dulbecco's Modified Eagle's Medium; FBS, fetal bovine serum. 
either MLL1 knockdown or MLL1 overexpression proceeded through the $S$ and $\mathrm{G}_{2} / \mathrm{M}$ phases with efficiencies and kinetics similar to those of control cells (Figure 2B, middle and right panels). However, after the release of the cells from $\mathrm{G}_{0} / \mathrm{G}_{1}$ arrest, knockdown of the expression of MLL1 with siRNA was associated with a decrease in the population of cells in the $\mathrm{S}+\mathrm{G}_{2} / \mathrm{M}$ phase and a concomitant increase in the population of cells in the $\mathrm{G}_{0} / \mathrm{G}_{1}$ phase (Figure 2B, left panel). Consistently, overexpression of MLL1 resulted in an accumulation of cells in the $\mathrm{S}+\mathrm{G}_{2} / \mathrm{M}$ phase. Similar results were also observed in $\mathrm{SiHa}$ cells (data not shown). Next, in order to confirm that the phenotypic of cells was specifically caused by MLL1 protein, we used MLL1-siRNA-resistant combination of MLL1 to transfect into HeLa cell. As shown in Figure 2C, the expression of MLL1 in HeLa cells when MLL1 was depleted led to an increase in the $\mathrm{S}+\mathrm{G}_{2} / \mathrm{M}$ phases, similar to the profile of the cells that co-transfected with nonspecific siRNA and combination of MLL1, suggesting that the phenotypic of cells was specifically caused by MLL1. Collectively, these results revealed that MLL1 promoted the proliferation of cervical carcinoma cells by promoting the $\mathrm{G}_{1} / \mathrm{S}$ transition.

\section{Identification of cyclin DI as a downstream target for MLLI}

MLL1 promoted proliferation of cervical carcinoma cells, but the detailed mechanism is still unknown. Cell cycle is regulated by numerous proteins, such as cyclin D1, ${ }^{20} \mathrm{p} 21,{ }^{21}$ $\mathrm{p} 27,{ }^{22}$ and cyclin E1. ${ }^{23}$

Consequently, we detected whether these proteins were regulated by MLL1. We overexpressed or silenced MLL1 in HeLa cells and observed that only cyclin D1 was obviously changed in mRNA and protein levels, while p21, p27, and cyclin E1 were not affected by MLL1 (Figure 3A). In order to further explore the MLL1-mediated cell proliferation, loss-of-function and gain-of-function experiments were performed in HeLa cells under the indicated experimental conditions, where the expression of MLL1 was silenced, while the four genes (CCND1, CCNE1, p21, and p27) were overexpressed individually, or where MLL1 was ectopically expressed, while the expression of the four genes (CCND1, CCNE1, p21, and p27) was individually silenced. We next utilized bromodeoxyuridine to measure the proliferation of HeLa cells under the indicated experimental conditions. As shown in Figure 3B (upper panel), both the loss-of-function and gain-of-function experiments demonstrated that $\mathrm{p} 21, \mathrm{p} 27$, and cyclin E1 had slight effects on cell proliferation, which were caused by MLL1. The strongest effect was caused by cyclin D1, suggesting that
CCND1 may be an important downstream target of MLL1 in regulating the cell proliferation activity. Western blotting was used to determine the protein level (Figure 3B, lower panel). Similar results could be seen in $\mathrm{SiHa}$ cells (data not shown). These results further confirm the argument that CCND1 gene is a downstream target for MLL1 in cervical carcinoma cells.

In order to further investigate the function of cyclin D1 in the proliferation of cervical carcinoma, which is MLL1 mediated, we tested whether overexpression of cyclin D1 could alleviate the effect of $\mathrm{G}_{0} / \mathrm{G}_{1}$ accumulation under MLL1 knockdown. For this purpose, HeLa cells were cotransfected with cyclin D1 expression construct and MLL1-siRNA or with an empty vector plus MLL1-siRNA as control. FACScan flow cytometry analysis showed that overexpression of cyclin D1 in HeLa cells when MLL1 was silenced resulted in a dramatic increase in cells in the $\mathrm{S}$ phase (Figure 3C, left panel). Interestingly, during knockdown of cyclin D1 expression, even with MLL1 overexpression, cells had a failure in the $\mathrm{G}_{1} / \mathrm{S}$ transition in HeLa cells (Figure 3C, left panel). The expression of MLL1 and cyclin D1 under different experimental conditions are shown in Figure 3C (right panel). These studies strongly favor the argument that cyclin D1 is a key downstream mediator of MLL1 in facilitating the $G_{1} / S$ transition and cell proliferation.

To determine whether MLL1 transcriptional regulated $C C N D 1$, we performed luciferase reporter assay to examine the effect of MLL1 on CCND1 promoter activity. As shown in Figure 3D, in both HeLa cells and SiHa cells, MLL1 transcriptional activated $C C N D 1$ gene promoter. Furthermore, chromatin immunoprecipitation assay also confirmed that MLL1 transcriptional regulated CCND1 (Figure 3E). These results demonstrated that MLL1 transcriptional activated $C C N D 1$.

\section{Physical and functional interactions between MLLI and $\beta$-catenin}

There were multiple studies that indicated that Wnt signaling pathway regulated $C C N D 1 ;{ }^{24}$ so we hypothesized whether MLL1 connects with the Wnt pathway in the transcriptional activation of $C C N D 1$. For this purpose, we transfected the $\beta$-catenin expression vector and the MLL1 expression vector either in combination or alone into HeLa cells, and then measured the $C C N D 1$ promoter-driven luciferase activity. As shown in Figure 4A, expression of either $\beta$-catenin or MLL1 alone was correlated with an increased CCND1 promoter activation, while cotransfection of $\beta$-catenin and MLL1 resulted in a synergistic effect. The efficiency 
of $\beta$-catenin siRNA was detected by Western blotting (Figure 4B). Moreover, we found that not only the CCND1 promoter diminished but also the synergy between $\beta$-catenin and MLL1 no longer existed in the $\beta$-catenin-silenced cells. In addition, when MLL1 was silenced, reporter activity due to $\beta$-catenin stimulation was also dramatically reduced.

So whether MLL1 interacted with $\beta$-catenin or not, we used MLL1 and $\beta$-catenin antibody to perform reciprocal co-immunoprecipitation and glutathione S-transferase assays. The results showed that MLL1 interacts with $\beta$-catenin (Figure $4 \mathrm{C}$ and D).

These results reveal that MLL1 is functionally correlated with $\beta$-catenin in the transcriptional activation of $C C N D 1$.
MLLI promotes cervical carcinoma cell metastasis through interaction with $\beta$-catenin

Cancer cell metastasis always resulted in poor prognosis. Wnt signaling pathway has been reported to be correlated with cancer cell metastasis. ${ }^{25}$ Our study also demonstrated that MLL1 interacted with $\beta$-catenin. Hence, we subsequently utilized transwell migration assays and anchorage-independent cell growth assays to explore the effect of MLL1 on migration of cancer cells.

We overexpressed MLL1 in highly metastatic cervical carcinoma cell lines C-33A and detected MLL1 expression in C-33A cells (Figure 5A). Transwell assay demonstrated
A

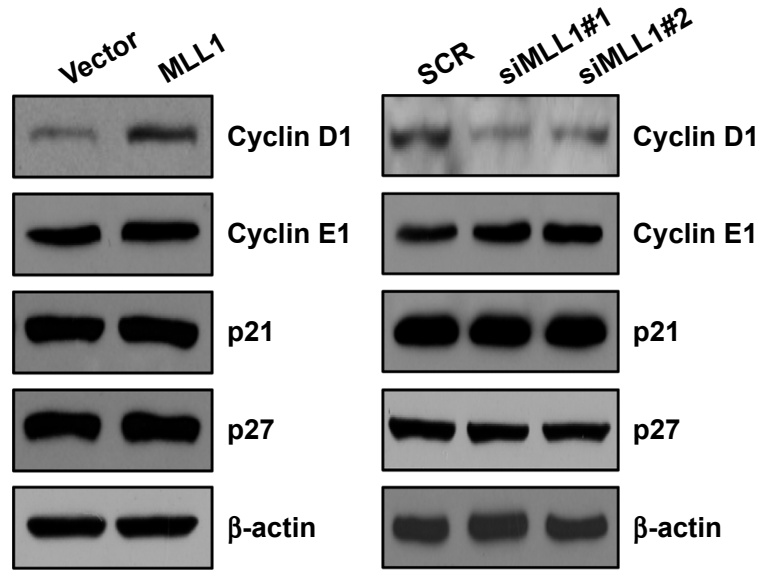

B

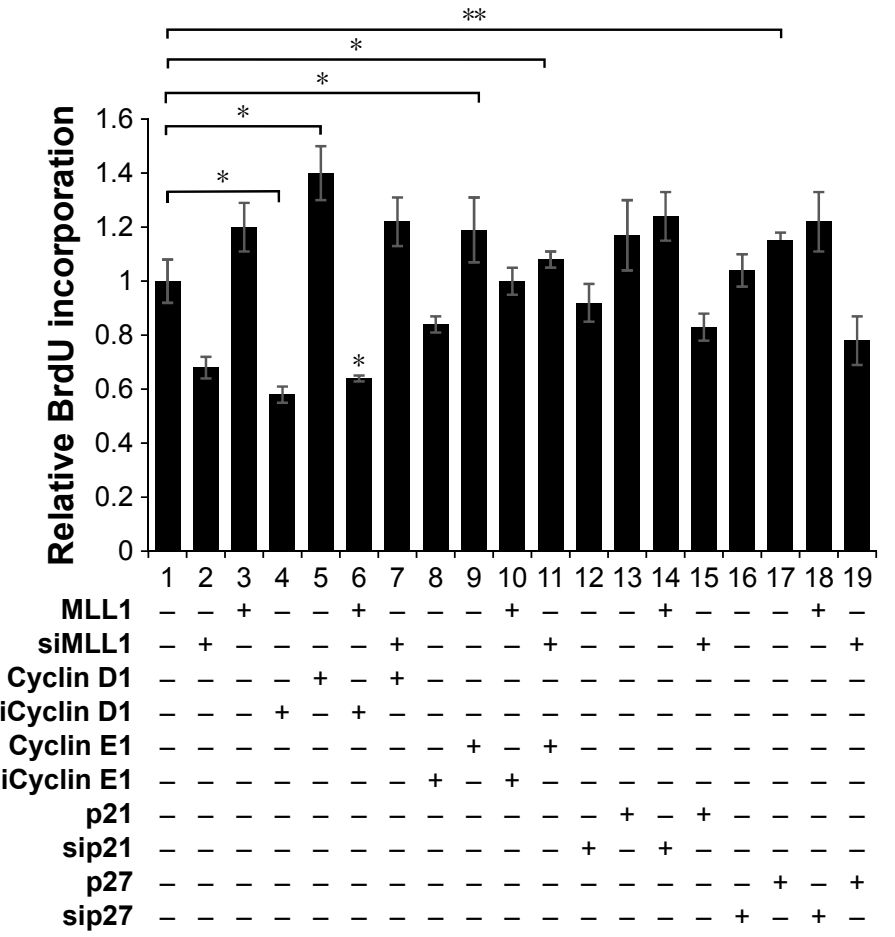

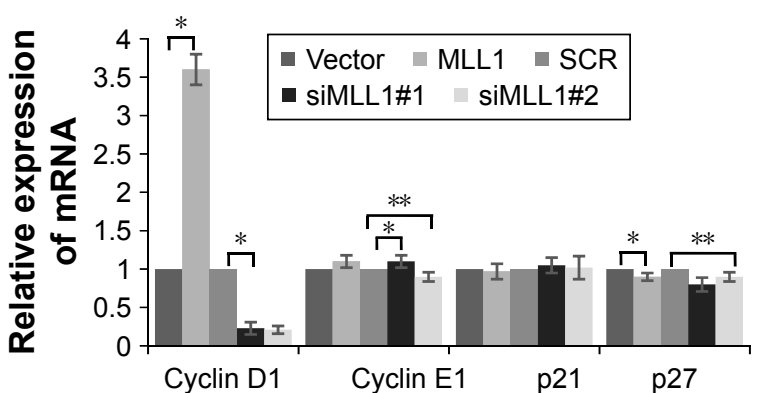
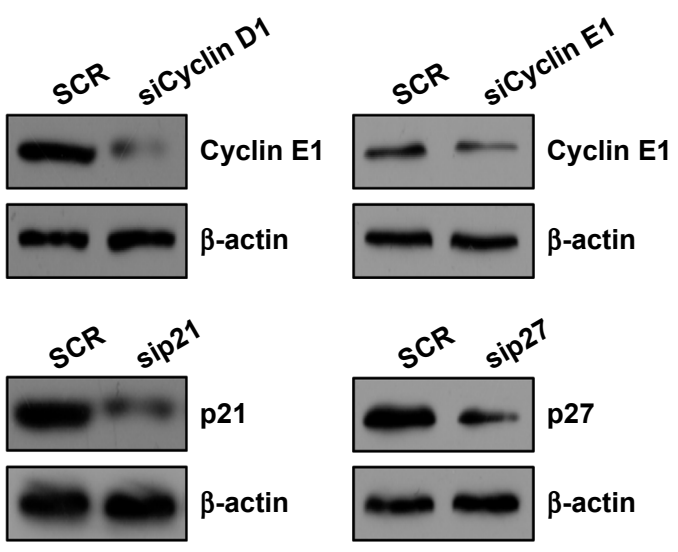

Figure 3 (Continued) 

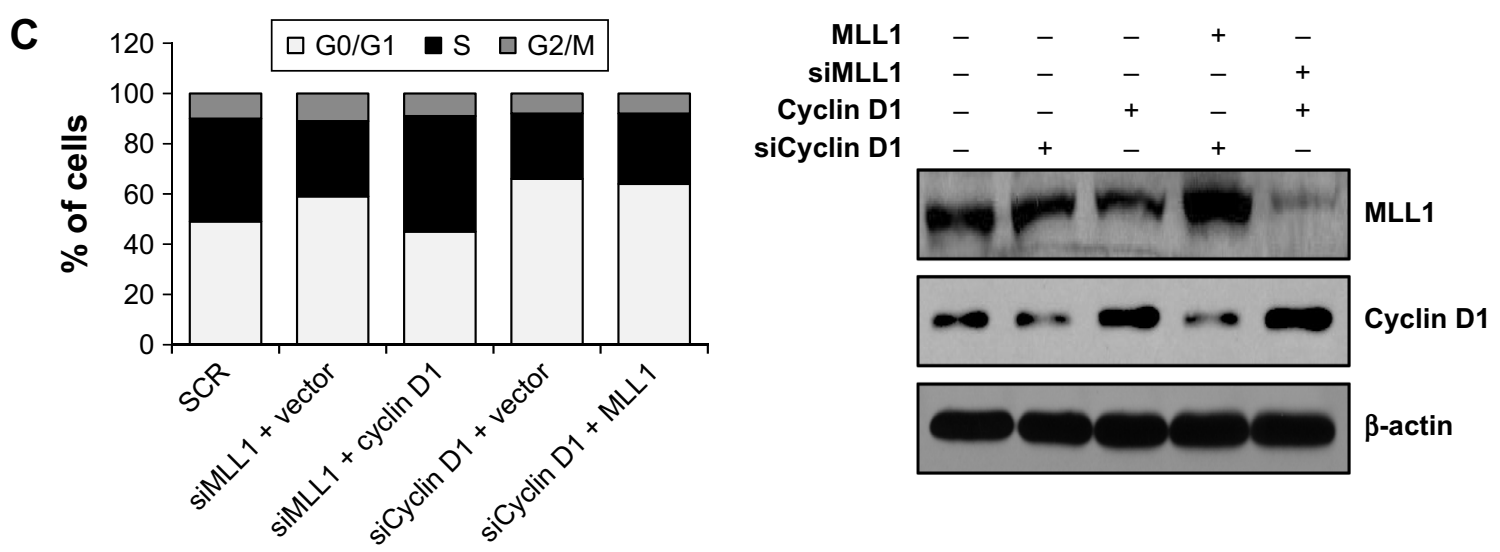

D

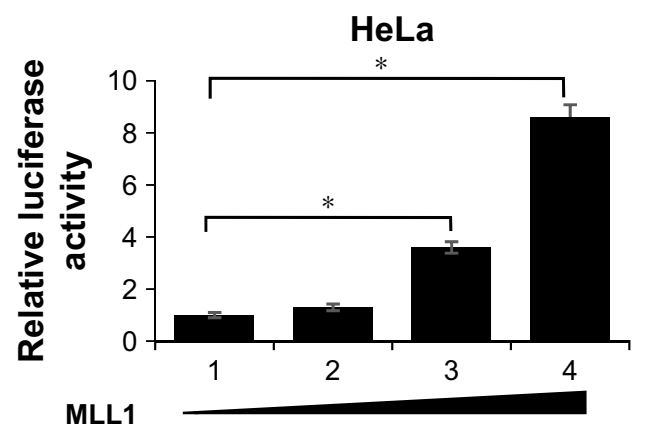

E Input $\lg G$

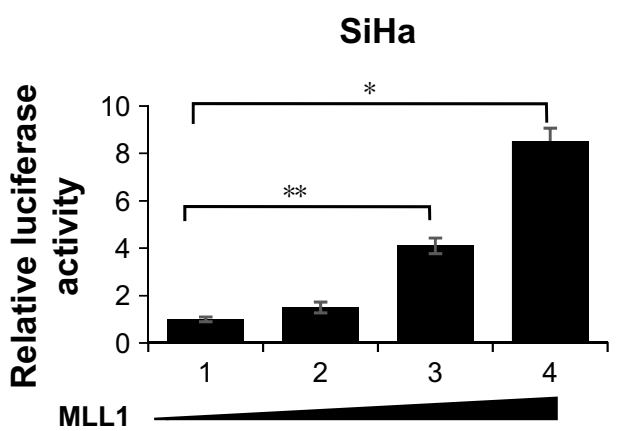

Cyclin D1

Figure 3 Identification of cyclin DI as a downstream target for MLLI.

Notes: (A) Upon overexpression and knockdown of MLLI, the expression of cyclin DI, cyclin EI, p2I, and p27 was detected, which are cell-cycle regulatory proteins. HeLa cells were transfected with FLAG-MLLI or MLLI-siRNA. Total proteins or RNA was extracted and examined by Western blotting or qRT-PCR analysis, respectively. Student's $t$-test was used to compare between two groups $(* P<0.05, * * P<0.01)$. (B) Effect of cyclin DI, cyclin EI, p2I, and p27 on MLLI-mediated cell proliferation. Over expressed or silenced MLLI, Cyclin DI, Cyclin EI, p2I and p27 in Hela cells. Each experiment was performed at least three times (upper panel). Western blotting was used to examine the expression of indicated proteins (lower panel). Student's $t$-test was used to compare between two groups ( $* P<0.05$, $* * P<0.01$ ). (C) Serum-starved HeLa cells were treated under indicated experimental conditions after 24 hours of transfection, and the cells were released by culturing in the DMEM medium with I0\% FBS for another 18 hours. Next, the cells were collected for cell-cycle profile analysis through flow cytometry. The percentage of cells in different cell-cycle phases are shown (left panel). Western blotting was used to examine the expression of indicated proteins (right panel). (D) HeLa (left) and SiHa (right) cells were cotransfected with the Renilla construct, CCNDI-Luc reporter, and different amounts of MLLI expression construct ( $50 \mathrm{ng} /$ well, $200 \mathrm{ng} /$ well, and $800 \mathrm{ng} /$ well). After 24 hours of transfection, the cells were harvested and luciferase reporter assay was performed. Each experiment was performed at least three times. The result indicated the recruitment of MLLI on the CCNDI promoter. Student's $t$-test was used to compare between two groups $(* P<0.05, * * P<0.01)$. (E) ChIP assay in HeLa cells with anti-MLLI or rabbit normal lgG was performed. The final DNA extraction was amplified by PCR using special primers in the promoter region of the CCNDI gene.

Abbreviations: qRT-PCR, quantitative reverse transcription polymerase chain reaction; BrdU, bromodeoxyuridine; DMEM, Dulbecco's Modified Eagle's Medium; FBS, fetal bovine serum; ChIP, chromatin immunoprecipitation.

that the percentage of invasive cells was strongly increased in cell when MLL1 was overexpressed, compared with the control group (Figure 5B). In order to investigate the function of $\beta$-catenin in the metastasis of cervical carcinoma mediated by MLL1, we tested whether overexpression of $\beta$-catenin could alleviate the effect of metastasis inhibition under MLL1 knockdown. For this purpose, C-33A cells were cotransfected with $\beta$-catenin expression construct and MLL1siRNA or with an empty vector plus MLL1-siRNA as control. The result showed that MLL1 depletion would reduce invade ability of C-33A cells; however, overexpression of $\beta$-catenin could alleviate the effect of MLL1 depletion (Figure 5C). The abovementioned experiments suggested that MLL1 would enhance the invasion ability of C-33A cells through interaction with $\beta$-catenin. Consequently, anchorage-independent cell growth assay was performed in the C-33A cells. As shown in Figure 5D, the silencing of MLL1 expression obviously inhibited the colony formation and overexpression of $\beta$-catenin could alleviate the effect of MLL1 depletion. All the results supported that MLL1 obviously stimulate cell migration through interaction with $\beta$-catenin.

\section{Discussion}

It is widely accepted that aberrant expression of transcription factors could affect the fate of a cell and proliferation generally caused carcinoma. The main characteristics of 
A

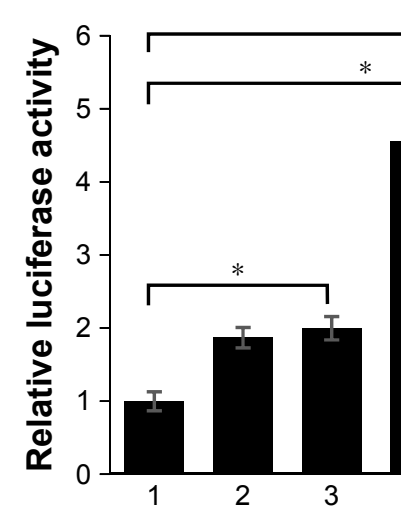

MLL1

$\beta$-catenin

siMLL1

si $\beta$-catenin

C

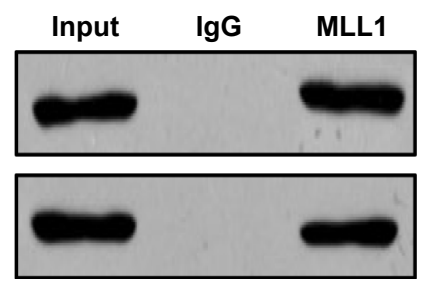

*
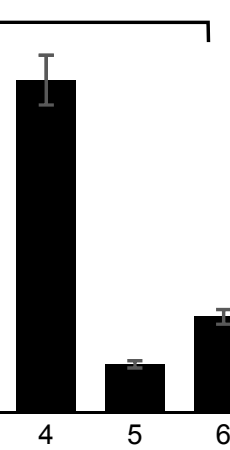

$-+$

- $\quad+$

- $-\quad-$

$-$
45
B
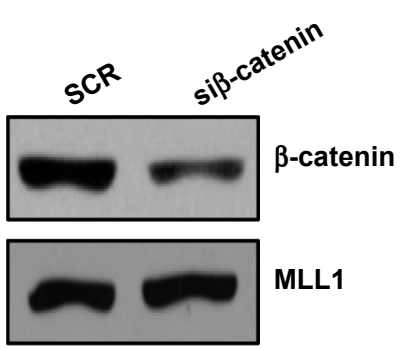

MLL1

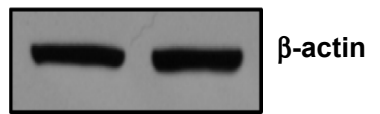

MLL1

D

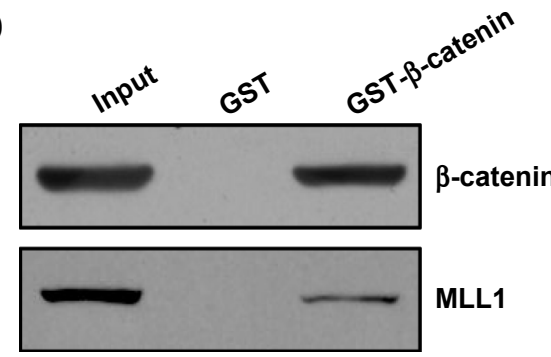

$\beta$-catenin

Figure 4 The physical and functional interactions between MLLI and $\beta$-catenin.

Notes: (A) $\beta$-catenin and MLLI synergistically transcriptional activate CCNDI. HeLa cells were cotransfected with the Renilla construct, CCNDI-Luc reporter, and the indicated expression constructs (MLLI $0.15 \mathrm{~g} /$ well, $\beta$-catenin $0.15 \mathrm{~g} /$ well). After 24 hours of transfection, the cells were collected and luciferase reporter assay was performed. Student's $t$-test was used to compare between two groups. Each experiment was performed at least three times (*P<0.05). (B) Efficiency of $\beta$-catenin siRNA was confirmed by Western blotting. (C) Co-IP results for MLLI and $\beta$-catenin. (D) GST pull-down results for MLLI and $\beta$-catenin.

Abbreviations: Co-IP, co-immunoprecipitation; GST, glutathione S-transferase.

human cervical carcinoma are rapid tumor cell proliferation, high invasiveness, and poor prognosis. Numerous genetic alterations and molecular abnormal regulation result in tumorigenesis of cervical carcinoma.
Through expression analysis of a panel of normal human cervical cell lines and cervical carcinoma cell lines, we showed that MLL1 is also overexpressed in human cervical carcinoma cell lines and tissues, and its expression levels are

\section{A}
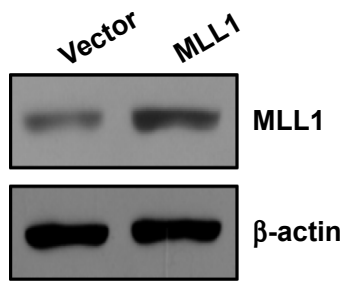

B

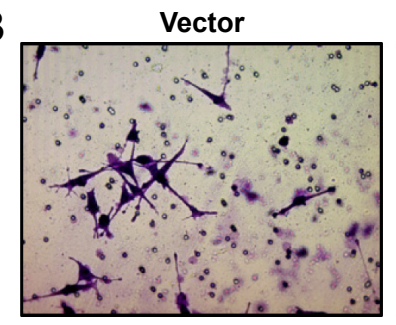

MLL1

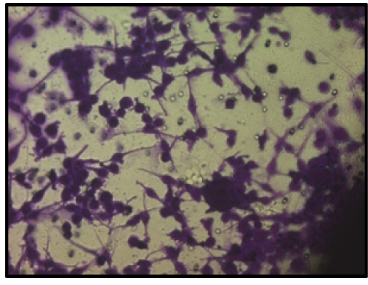

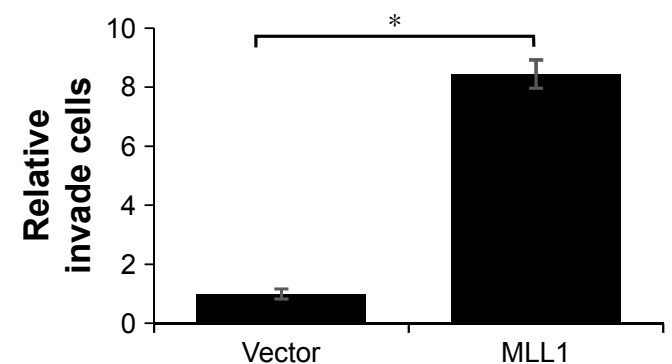

Figure 5 (Continued) 
C
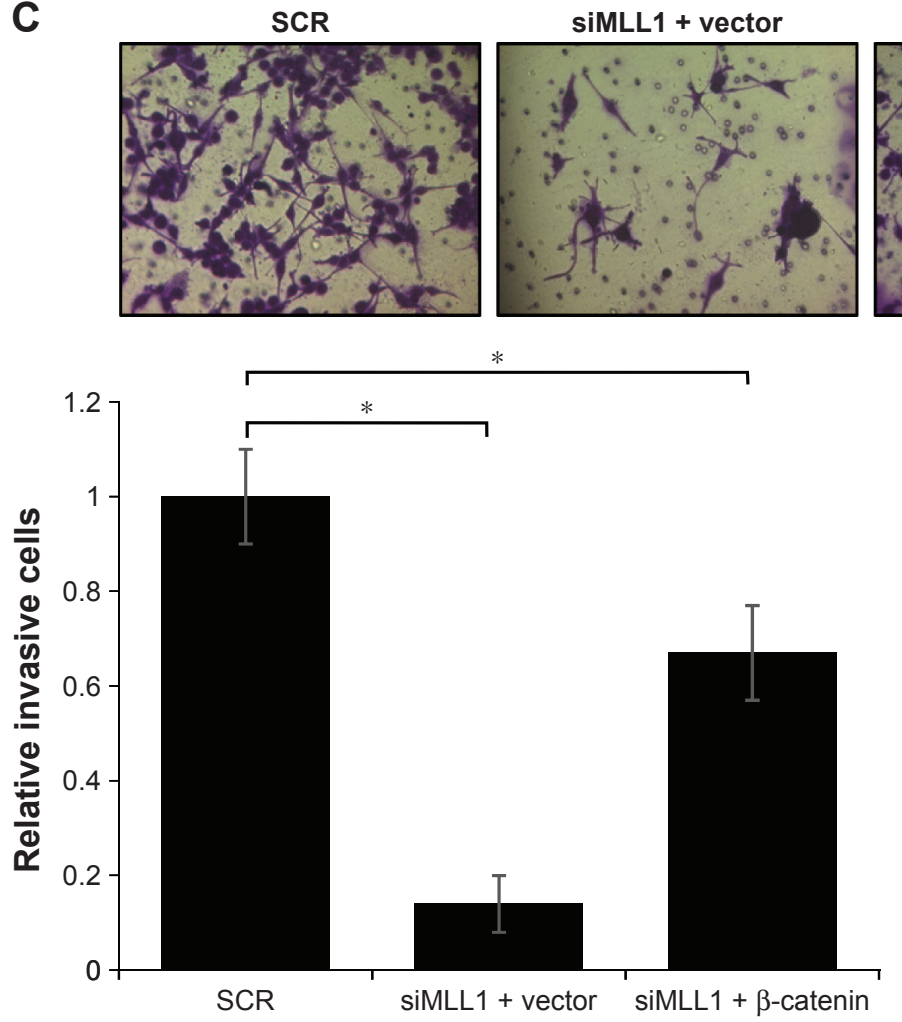

D

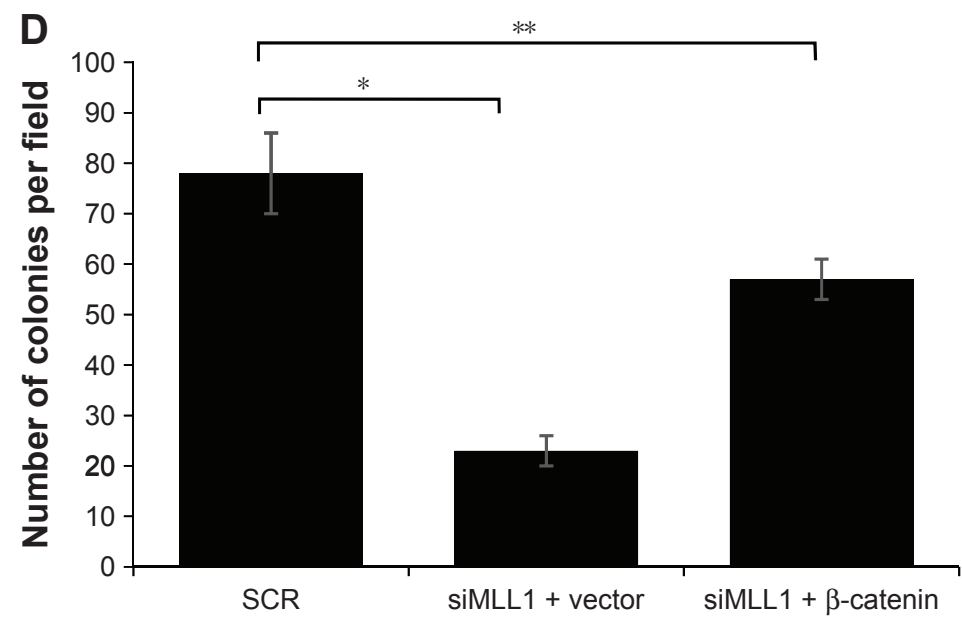

siMLL1 + $\beta$-catenin
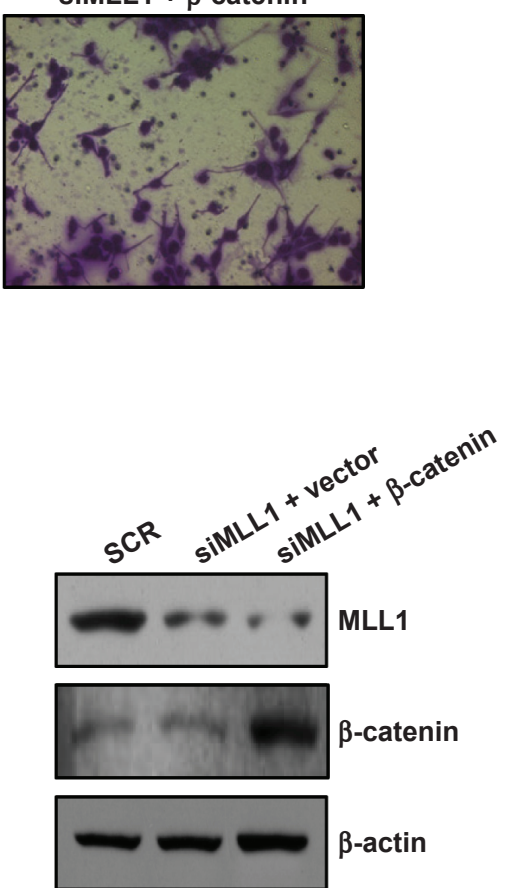

Figure $5 \mathrm{MLLI}$ promotes cervical carcinoma cell metastasis through interaction with $\beta$-catenin.

Notes: (A) The expression of MLLI in C-33A cells transfected with FLAG-MLLI was detected by Western blotting. (B) C-33A cells were transfected with MLLI and next subjected to invasion assay 48 hours later. Representative photos are shown. Student's $t$-test was used to compare between two groups. Each experiment was performed at least three times $(* P<0.05)$. (C) C-33A cells were transfected with MLLI-siRNA or MLLI-siRNA and $\beta$-catenin and next subjected to invasion assay 48 hours later. Representative photos are shown. Student's $t$-test was used to compare between two groups. Each experiment was performed at least three times ( $* P<0.05)$. The expression of MLLI and $\beta$-catenin in C-33A cells transfected with MLLI-siRNA or MLLI-siRNA and $\beta$-catenin was detected by Western blotting. (D) C-33A cells were transfected with MLLI-siRNA or MLLI-siRNA and $\beta$-catenin, and anchorage-independent growth ability of C-33A cells in soft agar was detected. Student's $t$-test was used to compare between two groups. Each experiment was performed at least three times $(* P<0.05 ; * * P<0.01)$.

related with tumor grade. Both loss-of-function and gainof-function experiments suggested that MLL1 contributed to proliferation of cervical carcinoma cells. At the cellular level, we found that MLL1 promoted cell-cycle progression by increasing the $\mathrm{G}_{1}$ to $\mathrm{S}$ transition.

MLL1 usually worked as a transcription factor. We next investigated the detailed mechanism of MLL1 in the regulation of cell cycle. Cell cycle is regulated by numerous proteins, such as CCND $1,{ }^{20} \mathrm{p} 21,{ }^{21} \mathrm{p} 27,{ }^{22}$ and CCNE1 ${ }^{23}$ Among these proteins, CCND1 has been demonstrated to be transcriptional activated by MLL1 transcription and to mediate the effect of MLL1 in promoting cell proliferation. Cyclin D1 is the product of CCND1, which is one of the pivotal regulators in the $\mathrm{G}_{0} / \mathrm{G}_{1}$ to $\mathrm{S}$ transition. We identified 
CCND1 as the downstream target of MLL1; this finding also agrees with the cellular behavior of FOXK2 in facilitating the $\mathrm{G}_{0} / \mathrm{G}_{1}$ to $\mathrm{S}$ transition.

In our study, we revealed that $\beta$-catenin acted synergistically with MLL1 in transcriptional activation of CCND1, and $\beta$-catenin and MLL1 were physically and functionally associated, indicating that $\beta$-catenin might be a transcription partner for MLL1 in cervical carcinoma.

\section{Conclusion}

We found that MLL1 is overexpressed in cervical carcinoma. We demonstrated that MLL1 was able to facilitate cell proliferation through promoting the $G_{1} / S$ transition of the cell cycle. We also identified CCND1 as a downstream target of MLL1. Furthermore, our study suggested that $\beta$-catenin functioned as the transcription partner of MLL1 in mediating CCND1 transcription. In addition, MLL1 promoted metastasis through interacting with $\beta$-catenin. Our experiments not only revealed a novel marker of cervical carcinoma but also found a new function for $\beta$-catenin in cervical carcinoma tumorigenesis.

\section{Disclosure}

The authors report no conflicts of interest in this work.

\section{References}

1. Mousavi-Jarrrahi SH, Kasaeian A, Mansori K, Ranjbaran M, Khodadost M, Mosavi-Jarrahi A. Addressing the younger age at onset in breast cancer patients in Asia: an age-period-cohort analysis of fifty years of quality data from the international agency for research on cancer. ISRN Oncol. 2013;2013:429862.

2. Coleman MP, Gatta G, Verdecchia A, et al. EUROCARE-3 summary: cancer survival in Europe at the end of the 20th century. Ann Oncol. 2003; 14(suppl 5):v128-v149.

3. Leegte B, Kerstjens-Frederikse WS, Deelstra K, Begeer JH, van Essen AJ. 11q-syndrome: three cases and a review of the literature. Genet Couns. 1999;10(3):305-313.

4. Canaani E, Nakamura T, Rozovskaia T, et al. ALL-1/MLL1, a homologue of Drosophila TRITHORAX, modifies chromatin and is directly involved in infant acute leukaemia. Br J Cancer. 2004;90(4):756-760.

5. Marschalek R. Mixed lineage leukemia: roles in human malignancies and potential therapy. FEBS J. 2010;277(8):1822-1831.

6. Milne TA, Briggs SD, Brock HW, et al. MLL targets SET domain methyltransferase activity to Hox gene promoters. Mol Cell. 2002;10(5): 1107-1117.

OncoTargets and Therapy

\section{Publish your work in this journal}

OncoTargets and Therapy is an international, peer-reviewed, open access journal focusing on the pathological basis of all cancers, potential targets for therapy and treatment protocols employed to improve the management of cancer patients. The journal also focuses on the impact of management programs and new therapeutic agents and protocols on
7. Yu BD, Hess JL, Horning SE, Brown GA, Korsmeyer SJ. Altered Hox expression and segmental identity in Mll-mutant mice. Nature. 1995; 378(6556):505-508.

8. Milne TA, Dou Y, Martin ME, Brock HW, Roeder RG, Hess JL. MLL associates specifically with a subset of transcriptionally active target genes. Proc Natl Acad Sci U S A. 2005;102(41):14765-14770.

9. Terranova R, Agherbi H, Boned A, Meresse S, Djabali M. Histone and DNA methylation defects at Hox genes in mice expressing a SET domain-truncated form of Mll. Proc Natl Acad Sci US A. 2006;103(17): 6629-6634.

10. Dey N, Barwick BG, Moreno CS, et al. Wnt signaling in triple negative breast cancer is associated with metastasis. BMC Cancer. 2013;13:537.

11. DiMeo TA, Anderson K, Phadke P, et al. A novel lung metastasis signature links Wnt signaling with cancer cell self-renewal and epithelial-mesenchymal transition in basal-like breast cancer. Cancer Res. 2009;69(13):5364-5373.

12. Lin SY, Xia W, Wang JC, et al. Beta-catenin, a novel prognostic marker for breast cancer: its roles in cyclin D1 expression and cancer progression. Proc Natl Acad Sci U S A. 2000;97(8):4262-4266.

13. Shang Y, Hu X, DiRenzo J, Lazar MA, Brown M. Cofactor dynamics and sufficiency in estrogen receptor-regulated transcription. Cell. 2000;103(6):843-852.

14. Shang Y, Brown M. Molecular determinants for the tissue specificity of SERMs. Science. 2002;295(5564):2465-2468.

15. Yin N, Wang D, Zhang H, et al. Molecular mechanisms involved in the growth stimulation of breast cancer cells by leptin. Cancer Res. 2004; 64(16):5870-5875.

16. Zhang H, Yi X, Sun X, et al. Differential gene regulation by the SRC family of coactivators. Genes Dev. 2004;18(14):1753-1765.

17. Wu H, Chen Y, Liang J, et al. Hypomethylation-linked activation of PAX2 mediates tamoxifen-stimulated endometrial carcinogenesis. Nature. 2005;438(7070):981-987.

18. Zhang H, Sun L, Liang J, et al. The catalytic subunit of the proteasome is engaged in the entire process of estrogen receptor-regulated transcription. EMBO J. 2006;25(18):4223-4233.

19. Shifrin VI, Davis RJ, Neel BG. Phosphorylation of protein-tyrosine phosphatase PTP-1B on identical sites suggests activation of a common signaling pathway during mitosis and stress response in mammalian cells. J Biol Chem. 1997;272(5):2957-2962.

20. Lebeau A, Unholzer A, Amann G, et al. EGFR, HER-2/neu, cyclin D1, p21 and p53 in correlation to cell proliferation and steroid hormone receptor status in ductal carcinoma in situ of the breast. Breast Cancer Res Treat. 2003;79(2):187-198.

21. Karimian A, Ahmadi Y, Yousefi B. Multiple functions of p21 in cell cycle, apoptosis and transcriptional regulation after DNA damage. DNA Repair. 2016;42:63-71.

22. Das RK, Huang Y, Phillips AH, Kriwacki RW, Pappu RV. Cryptic sequence features within the disordered protein $\mathrm{p} 27 \mathrm{Kip} 1$ regulate cell cycle signaling. Proc Natl Acad Sci U S A. 2016;113(20):5616-5621.

23. Sauer K, Lehner CF. The role of cyclin $\mathrm{E}$ in the regulation of entry into S phase. Prog Cell Cycle Res. 1995;1:125-139.

24. Tetsu O, McCormick F. Beta-catenin regulates expression of cyclin D1 in colon carcinoma cells. Nature. 1999;398(6726):422-426.

25. Li Y, Jin K, van Pelt GW, et al. c-Myb enhances breast cancer invasion and metastasis through the Wnt/beta-catenin/Axin2 pathway. Cancer Res. 2016;76(11):3364-3375.

\section{Dovepress}

patient perspectives such as quality of life, adherence and satisfaction The manuscript management system is completely online and includes a very quick and fair peer-review system, which is all easy to use. Visit http://www.dovepress.com/testimonials.php to read real quotes from published authors. 